\title{
Efficacy and safety of superficial chemical peeling in treatment of active acne vulgaris*
}

\author{
Hassanain Al-Talib ${ }^{1}$ \\ Ayad Hameed ${ }^{3}$
}

\author{
Alyaa Al-khateeb ${ }^{2}$ \\ Chandrika Murugaiah ${ }^{4}$
}

DOI: http:/ / dx.doi.org/10.1590/abd1806-4841.20175273

\begin{abstract}
Acne vulgaris is an extremely common condition affecting the pilosebaceous unit of the skin and characterized by presence of comedones, papules, pustules, nodules, cysts, which might result in permanent scars. Acne vulgaris commonly involve adolescents and young age groups. Active acne vulgaris is usually associated with several complications like hyper or hypopigmentation, scar formation and skin disfigurement. Previous studies have targeted the efficiency and safety of local and systemic agents in the treatment of active acne vulgaris. Superficial chemical peeling is a skin-wounding procedure which might cause some potentially undesirable adverse events. This study was conducted to review the efficacy and safety of superficial chemical peeling in the treatment of active acne vulgaris. It is a structured review of an earlier seven articles meeting the inclusion and exclusion criteria. The clinical assessments were based on pretreatment and posttreatment comparisons and the role of superficial chemical peeling in reduction of papules, pustules and comedones in active acne vulgaris. This study showed that almost all patients tolerated well the chemical peeling procedures despite a mild discomfort, burning, irritation and erythema have been reported; also the incidence of major adverse events was very low and easily manageable. In conclusion, chemical peeling with glycolic acid is a well-tolerated and safe treatment modality in active acne vulgaris while salicylic acid peels is a more convenient for treatment of darker skin patients and it showed significant and earlier improvement than glycolic acid
\end{abstract}

Keywords: Acne vulgaris; Salicylic acid; Polyglycolic acid

\section{INTRODUCTION}

Acne vulgaris is a chronic disorder of the pilosebaceous apparatus caused by abnormal desquamation of follicular epithelium. This leads to the obstruction of the pilosebaceous canal, resulting in inflammation and subsequently the formation of papules, pustules, nodules, comedones, and scarring. ${ }^{1}$ Acne vulgaris can greatly influence a person's quality of life therefore, it is necessary to deal with acne vulgaris to prevent emotional consequences and persistent complications. ${ }^{2}$ Acne vulgaris is the most common skin disease among teenagers in the US. About $85 \%$ of general population has an acne vulgaris in their lives, which mostly included on the face, upper back and chest. ${ }^{3}$ Acne vulgaris is caused by over activity of the sebaceous glands and blockage in the ducts, which leads to the formation of comedones that later on inflamed, by Propionibacterium acnes. ${ }^{3}$ Recently, the chemical peel procedures are more commonly used in photo-aging, active acne vulgaris, actinic keratosis, and acne scar. ${ }^{4}$ Many clinicians propose the use of superficial chemical peels as a treatment option of acne vulgaris as it is a relatively safe procedure. ${ }^{4}$ Superficial chemical peels remains the standard for dealing with acne scar, fine lines, pigmentation and other skin problems.
Currently, superficial chemical peeling processes are widely used in treatment of active acne vulgaris and as daily simple procedure done by plastics surgeons or dermatologist in a therapeutic setting. ${ }^{5}$ Different superficial chemical peels are used in skin via removing of stratum corneum layer, smoothing out the rough area, promoting product ingredient penetration and enhancing collagen production. ${ }^{6}$ Using superficial chemical peels in treatment of active acne will breakdown corneosomes, exfoliate superficial layers of epidermis, increase epidermal activity of enzymes and lead to epidermolysis, in addition to deal with a percentage of the current lesions. ${ }^{7}$ Acne vulgaris is commonly associated with stress, anxiety, high humidity, exposure to dirt, hormonal changes, and drugs. ${ }^{5}$ Many regimens are used for its treatment like topical retinoids, benzoyl peroxide, topical antibiotic, oral antibiotics and hormone therapy. ${ }^{5}$ Limited studies have been conducted on using superficial chemical peels on patients with active acne vulgaris and the efficacy and safety of such treatment options are yet to be determined through the review of the clinical results. Although the topical treatments are effective, other acne cases like active acne and resistant acne vulgaris are hard 
to treat. The use of chemical peels agents to manage such conditions should be focused to achieve effective and safe clinical outcomes. The purpose of this study was to review the efficacy of superficial chemical peeling in the treatment of active acne vulgaris and to determine the safety of superficial chemical peeling in the treatment of active acne vulgaris.

\section{METHODOLOGY}

Abstracts of previously published articles with titles relating to the study topics were viewed and relevant articles were downloaded and viewed for details. Literatures selection was done based on inclusion and exclusion criteria. Manuscripts which identify the efficiency and safety superficial chemical peeling in the treatment of active acne vulgaris were chosen to be used for the structured review. Quality Assessment checks on the papers, research table was constructed, the findings were compared and interpreted and discussions and conclusion were made.

\section{BIBLIOGRAPHIC DATABASES}

A literature search was conducted both electronically and manually. Electronic data and articles published in the English language were searched online in PubMed. Manual search was also done on references from selected articles.

\section{LITERATURE SEARCH METHOD}

The studies included in the review were chosen according to the following elements: Patients $(\mathrm{P})$ we have included patients with active acne vulgaris; Intervention (I) using superficial chemical peels for active acne vulgaris; Comparison (C) Quantitative assessment of control and side effect of active acne.; Outcomes (O) Control and remission of active acne; Study (S) the study include prospective clinical trial. We conducted a search on PubMed and Google scholar using the terms "Active acne vulgaris, Glycolic acid (GA), Jessner's solution, Patients' number, Peeling, Peels, Salicylic acid (SA), Seborrhea, Superficial chemical peel, Trichloroacetic acid, Resorcinol." All prospective clinical trials or case series from 2000 and 2014 and written in English language which deal with human being only, were included and grouped according to the peeling agent used and the indication, being acne or acne scars. However studies that were excluded from this study were those without defining clinical events, also papers published in none peered review journals and those who neglecting certain sources and result in reviews being biased.

\section{QUALITY ASSESSMENT AND DATA EXTRACTION}

This study used JADED scoring for quality assessment for randomized controlled trial and Newcastle Ottawa for non-randomized controlled trial. Studies with the score 0-2 were considered to have a low quality and studies with the score $3-5$ were considered to have high quality. Data were extracted from the selected literatures using research table. The name of the first author, year of publication, journal name and the study title were captured. Sample size, duration of the study, patient, intervention, comparison, outcomes and study design (PICOs) of the reviewed papers were also included. Pre-procedure and post-procedure cares, types and parameters of superficial chemical peels used in the studies, assessment methods, outcomes and complications of the studies were summarized in the tables and analyzed.

\section{RESULTS}

A total of 32,556 articles were found in the literature search using the keywords and additional filters. Abstracts of 27 articles related to the study topics were viewed. Full text articles were downloaded. A total of 7 articles that met the inclusion and exclusion criteria were chosen to be used in this study. We found a total of seven articles on both superficial chemical peeling and active acne vulgaris whether on PubMed or Google scholar table $1 .{ }^{1}$ Among the seven articles on acne scars, one randomized prospective trial of split-face model and compared between GA and Jessner's solution, and showed no significant difference between two peels. ${ }^{8}$

There were two open label trials that evaluated the effects of SA $30 \%$ peels on comedones. ${ }^{9,10}$ One study evaluated a polyethylene glycol (PEG) vehicle that reduced the stinging sensation. Both studies revealed a significant improvement of both comedonal and acne lesions in greater than $75 \%$ of patients, with few or mild adverse events (Table 2). Other two studies evaluated the efficacy of GA on comedonic acne and showed improvement in retentional lesions after 3 months (Table 3). ${ }^{11,12}$ Both studies confirmed that a minimal adverse event was reported on $97 \%$ of patients who showed no any complaints. A study by Kessler et al found that both GA and SA were similarly effective in treatment of acne vulgaris with minimal adverse events reported by SA. ${ }^{13}$ There were two studies comparing GA and SA chemical peels, in first study SA had a higher efficacy than GA on acne lesions $(\mathrm{P}<0.001)$ with adverse events being more common with GA ( $40 \%)$ than with SA $(24 \%)$. Also SA peels for comedones and papules showed a significant improvement 4 weeks earlier than GA peels. However, in a second study there were no significant differences in effectiveness between GA and SA. ${ }^{14,15}$

\section{DISCUSSION}

Acne vulgaris is a common skin disease that affects young and old individuals and varies in types and severity. Acne vulgaris is treatable skin lesion. This study was conducted to review the useful effects of superficial chemical peels in treatment of active acne vulgaris. This study revealed that superficial chemical peels were

\begin{tabular}{llll}
\multicolumn{2}{c}{ TABLE 1: Summary of literature search } \\
\hline Database & Key words & No. of abstract retrieved & No. of an article selected \\
\hline PubMed & (1) Active acne vulgaris & 219 & - \\
& (2) Superficial chemical peels & 65 & 4 \\
Google Scholar & (1) e (2) & 2 & 1 \\
& (1) Active acne vulgaris & 14.700 & - \\
& (2) Superficial chemical peels & 16.400 & - \\
& (1) e (2) & 1.170 & 2
\end{tabular}




\section{TABLE 2: Study population with efficacy and adverse effects of chemical peels}

\section{Author} Population and acne Characteristics

Kessler, et $\quad \mathrm{N}=20$, American patients, (13-35)

al., 2008 (13). year, with Acne vulgaris mild to moderate inflamed pimple, two months duration study.

\section{Outcome of study}

Both GA and SA peels were significant ly effective by the second treatment and there were no significant differences in effectiveness between the two peels. SA peel showed sustained effectiveness two months post-treatment. More adverse events were reported with the GA peel. years with mostly severity acne,
Hashimoto $\mathrm{N}=16$, Japanese patients, (16-29) et al.,2008 (10). comedonal acne (less than (10) papules or pustules), Medications allowed, two and half months duration study.

Dainichi et $\quad \mathrm{N}=436$, Japanese patient, (17-46) al.,2008 (9). years, patients having comedonal acne, nodulo-cystic acne, concomitant acne and redness. Three months study period.

Garg et al. 2009 (14).

$\mathrm{N}=44$, Indian patients, $(16-27)$ years, with acne vulgaris and post-acne scarring and hyperpigmentation. Treated for 24 week.

Comedones are reduced to $75 \%$. The mean total facial comedones count, was decreased from 39.3 at baseline to 9.2 after 10 weeks treatment by the time of completion of the study.

Comedones are significant improvement (greater than $75 \%$ clearance of lesions oc curred).

Scanning electron microscopy after 1 week treatment with $30 \%$ SA revealed a restoration of the regular grooves of the skin and removal of the cornified plugs from the hair follicles.

Both GA and SA peels decrease the comedones; SA had a higher efficacy for most active acne lesions $(\mathrm{P}<0.001)$ and hyperpigmentation $(\mathrm{P}<0.001)$

SA peels for comedones and papules showed a significant improvement 4 weeks earlier than GA.

Dréno et al., $\mathrm{N}=397$, pacientes com (30-40) Inflammatory and retentional lesions were 2011(15). anos com pele de fototipos (II-IV) e estudo com duração de 3 meses.
Both open and closed comedones decrease significantly during study period.

\section{Tolerability}

Safety peeling for both GA and SA, mild complications increased after the first treatment and decreasing after the frequent treatments.

Mild burning sensation and erythema were noted in $8.8 \%$ of patients treated with SA.

No complaints of pain, burning, or stinging and no signs of edema, bleeding, crusting, or post-inflammatory pigmentation.

Adverse effects were less common with SA $(24 \%$ than GA with $40 \%$. Also $17.3 \%$ patients developed a burning or stinging sensation. In treatment with GA peels $8.7 \%$ of the patients had visible desquamation. Dryness was seen more often with SA treatment $(14.28 \%)$. Photosensitivity and initial acne flare were same among both GA and SA groups.

Adverse effects were infrequent with some patients complaint of irritation, tightening, pruritus, and $96.7 \%$ of the patients did not encounter any adverse effect.

More adverse effects seen by Jessner's solution as erythema and exfoliation form compared to GA $(p<0.01)$.

ty of acne had not changed among most of the patients. But after the third session, 50 $\%$ of the patients treated with either GA or Jessner's solution showed improvement. No significant differences in treatment effects between the 2 methods.

Atzori et al., $\mathrm{N}=80$, Italian patients $(16-27)$ Rapid improvement was observed in come1999 (11). years, with comedonal acne, nodu- donic acne within three applications of GA. lo-cystic and papulo-pustular con- In the papulo-pustular significant improvecomitant acne. Study period for 2 ment was achieved by the fourth application. years.
A minimal inflammatory lesions developed in $20 \%$ of the patients. one patient had tenacious erythema. Nodular-cystic forms required eight to ten applications. safe and effective. Peeling outcomes is dependent on many factors such as patient condition, stage of acne, peeling agent and its concentration, preparation before operation and follow up after operation as well as the skillful level of aesthetician or dermatologist. ${ }^{16}$ It is easy to achieve great chemical peel results without complications when appropriate pre-peel and post-peel consideration was contemplated.

Many chemical peels used in treatment of acne vulgaris like GA that is also used for the reduction of wrinkles. ${ }^{15}$ Although a slight redness and swelling might be seen after treatment with
GA and it's especially happens in black-skinned patients. ${ }^{5,17} \mathrm{GA}$ is an alpha-hydroxyl acid and works by removing the corneum stratum layer, scattering basal melanocytic layer and stimulating epidermolysis. ${ }^{18}$ Treatment with GA is generally tolerated and patient compliance is excellent, although GA showed no poisonous effect and has a long time span it should be avoided in contact dermatitis, pregnancy and lactation. ${ }^{18}$ Previous studies have indicated that GA peeling was affected by dose and duration of application with high concentration was more harmful on tissue than lower concentration. ${ }^{19}$ 


\title{
TABLE 3: Chemical peeling and post - peeling assessments
}

\author{
Estudo Peeling agents and Study design and post-peeling assessment \\ concentrations \\ Kessler, et GA $30 \%$, SA $30 \%$ \\ al., 2008 (13) \\ Double-blind, randomized controlled study, prospective, split-face. \\ Quantitative and qualitative assessment was done based on the number of papules and pustules \\ Clinical response was graded as: \\ Good > 50\% improvement. \\ Satisfactory $(21 \%-50 \%)$ \\ Poor $(10 \%-20 \%)$ \\ No change \\ Worsening

$\begin{aligned} & \text { Hashimoto } \\ & \text { et al.,2008 } \\ & \text { (10) }\end{aligned}$
$\begin{aligned} & \text { Dainichi et } 30 \% \\ & \text { al.,2008 (9) }\end{aligned}$ \\ Open-label trial, ten weeks study. \\ Clinical response was assessed by both changes in severity and comedone count reduction rate. \\ Open-label trial, non-specific peeling number, \\ Assessments by a questionnaire on adverse effects and efficacy of the peeling, which was filled \\ out by patients. The evaluation scores for each question were divided into five answer categories: \\ Not at all: $0 \%$. \\ Little: $0 \%$ to $25 \%$. \\ Some: $25 \%$ to $50 \%$ \\ Quite a lot: $50 \%$ to $75 \%$. \\ Very much: $75 \%$ to $100 \%$. \\ Garg et al., GA 35\%, SA 20\% -man- \\ 2009 (14) delic acid $10 \%$ peels \\ Open-label trial, comparative study. \\ Pre- treatment assessments of acne lesion was done by Michaelson method, \\ Post- treatment assessment of the response on a 5-point visual analog scale: \\ Good > 60\%. \\ Satisfactory $31-60 \%$. \\ Poor $<30 \%$ \\ No change \\ Worsening \\ Dréno et al., RAL $0.1 \%$, GA $6 \%$ \\ Open-label trial, \\ Efficacy of treatment was assessed by counting inflammatory lesions (papules \& pustules) and \\ retentional lesions (open and closed comedones) on days 30 and 90 using a 5- grade rating scale: \\ $0=$ no improvement. \\ $1=$ mild improvement. \\ $2=$ moderate improvement. \\ $3=$ notable improvement. \\ $4=$ very notable improvement .
}

Kim et al., GA 70\%, Jessner's solu- A randomized prospective clinical trial of split-face model therapy was done.

1999 (8) tion Skin lesions were graded by using Cunliffe's acne grading system based on depth and width of acne lesions.

0.25: a few small inflamed lesions.

0.5: small inflammatory lesions over a wider area.

1.0: more intensely inflamed.

1.5: intensely inflamed lesions over a wider area.

2.0: deeper lesions but not nodular.

Atzori et al., GA $70 \%$ $1999(11)$

Open-label trial, the procedure was well tolerated and patient compliance was excellent when asked to evaluate their skin.

Assessments by patient opinion and clinical rating based on this scale:

Mild improvement.

Moderate improvement.

Marked improvement.

Worsening.

$95 \%$ of the patients reported marked improvement, while the rest reported a moderate effect.

SA-PEG : Salicylic acid in polyethylene glycol, GA; Glycolic acid, RAL; Retinaldehyde, SA; Salicylic acid.

A study by Kim et al. confirmed that GA is superior to Jessner's solution in treatment active acne with less adverse events (erythema and exfoliation) compared with Jessner's solution $(\mathrm{p}<0.01)$. ${ }^{9}$ However, Garg et al found that GA had a lower efficacy than SA in treatment of active acne vulgaris $(\mathrm{P}<0.001)$ and hyperpigmenta- tion $(\mathrm{P}<0.001)$ since GA don't have anti-inflammatory effects hence making it lower rank to SA in the treatment of acne lesions. ${ }^{14}$ Another chemical peel used in treatment of acne vulgaris is SA which is a Beta-hydroxyl acid with both keratolytic properties and anti-inflammatory also could infiltrate simply to pilosebaceous layers and 
is not toxic, self-neutralizing with minimal dermal infiltration. ${ }^{20}$ Previous study showed that SA can be used for all stages of active acne due to its anti-inflammatory and comedolytic properties. ${ }^{20}$ The adverse events of salicylic acid are minor and temporary like dryness and erythema. Salicylic acid toxicity (Salicylism) occurs when applied to large area due to systemic absorption of the acid and is usually associated with high concentrations. ${ }^{20}$

Other chemical peeling agent Trichloroacetic acid (TCA) is an inorganic complex that could be applied alone or in combination with other chemical agents. ${ }^{21}$ In addition TCA causes epidermal necrosis and accelerate cellular growth. The TCA peeling endpoint is identified by a white frosted appearance on the skin, and then the process should be stopped at once. A lighter concentration of the peeling agent should be used in skin group type IV-VI. Frosting in these skin group types is not favored and may induce post-peel dyschromias and dark circle. ${ }^{17}$ TCA is highly effective agent in the treatment of active acne and skin pigmentation and it is steady, easy to set up and effectively accessible. TCA has some disadvantages like post-inflammatory hypopigmentation and post-inflammatory hyperpigmentation, which might be observed more commonly in higher concentrations and in dark-skinned patients. ${ }^{17,22}$
In summary, acne vulgaris is a chronic, relapsing disease. It is important to have an option for patients who may require a more aggressive treatment option or when the patient cannot tolerate common acne medications. Previous studies confirmed that, superficial chemical peel therapy played important role in reduction of papules, pustules and comedones in active acne vulgaris. Although, it was associated with minor adverse events like erythema, edema and occurrence of post-inflammatory hyperpigmentation or hypopigmentation, but such complications are transient and can be successfully managed. As Handog et al stated "Compared to newer machine based technologies for acne and acne scars, chemical peeling is affordable and with minimal downtime, and can be performed in any dermatologist's office" ${ }^{\prime 23}$

After reviewing previous studies it could be concluded that the both GA (30 - $70 \%)$ and SA (30 \%) were effective in reducing inflammatory lesions of acne. Also GA is highly tolerated and safer in treatment of acne among darker skin patients and could be used in treatment of acne scars as well. Moreover SA peels is more convenient for treatment patients with hyperpigmentation or darker skin due to its whitening efficacy on the skin even SA peels for comedones and papules showed a significant improvement earlier than GA. $\square$

\section{REFERENCES}

1. Thiboutot D, Gollnick H, Bettoli V, Dreno B, Kang S, Leyden JJ, et al. New insights into the management of acne: an update from the Global Alliance to Improve Outcomes in Acne group. J Am Acad Dermatol. 2009;60:S1-50.

2. Roberts WE. Chemical peeling in ethnic/dark skin. Dermatol Ther. 2004;17:196-205

3. Roberts JA, Uetz GW. Chemical signaling in a wolf spider: a test of ethospecies discrimination. J Chem Ecol. 2004;30:1271-84.

4. Markowitz PI.linventor Topical anionic salicylate for disorders of the skin. USA patent 6,120,756 2000.

5. Nast A, Bayerl C, Borelli C, Degitz K, Dirschka T, Erdmann R, et al. S2k-guideline for therapy of acne. J Dtsch Dermatol Ges. 2010; 8 Suppl 2:s1-59.

6. Daza MF, Jemec GBE. Glossary of Dermatological Terms. In: Ximena Wortsman, Jemec GBE, editors. Dermatologic Ultrasound With Clinical And Histologic Correlations. New York: Heidelberg Dordrecht London Springer; 2013. p. 573.

7. Deplewski D, Rosenfield RL. Role of hormones in pilosebaceous unit development. Endocr Rev. 2000;21:363-92.

8. Kim SW, Moon SE, Kim JA, Eun HC. Glycolic acid versus Jessner's solution: which is better for facial acne patients? A randomized prospective clinical trial of split-face model therapy. Dermatol Surg. 1999;25:270-3.

9. Dainichi T, Ueda S, Imayama S, Furue M. Excellent clinical results with a new preparation for chemical peeling in acne: $30 \%$ salicylic acid in polyethylene glycol vehicle. Dermatol Surg. 2008:34:891-9.

10. Hashimoto Y, Suga Y, Mizuno Y, Hasegawa T, Matsuba S, Ikeda S, et al. Salicylic acid peels in polyethylene glycol vehicle for the treatment of comedogenic acne in Japanese patients. Dermatol Surg. 2008:34:276-9

11. Atzori L, Brundu MA, Orru A, Biggio P.. Glycolic acid peeling in the treatment of acne. J Eur Acad Dermatol Venereol. 1999;12:119-22.

12. Dréno B, Fischer TC, Perosino E, Poli F, Viera MS, Rendon MI, et al. Expert opinion: efficacy of superficial chemical peels in active acne management--what can we learn from the literature today? Evidence-based recommendations. J Eur Acad Dermatol Venereol. 2011;25:695-704.

13. Kessler E, Flanagan K, Chia C, Rogers C, Glaser DA. Comparison of alpha- and beta-hydroxy acid chemical peels in the treatment of mild to moderately severe facial acne vulgaris. Dermatol Surg. 2008;34:45-50.
14. Garg VK, Sinha S, Sarkar R. Glycolic acid peels versus salicylic-mandelic acid peels in active acne vulgaris and post-acne scarring and hyperpigmentation: a comparative study. Dermatol Surg. 2009;35:59-65.

15. Dreno B, Castell A, Tsankov N, Lipozencic J, Serdaroglu S, Gutierrez V, et al. Interest of the association retinaldehyde/glycolic acid in adult acne. J Eur Acad Dermatol Venereol. 2009;23:529-32.

16. Rendon MI, Berson DS, Cohen JL, Roberts WE, Starker I, Wang B. Evidence and considerations in the application of chemical peels in skin disorders and aesthetic resurfacing. J Clin Aesthet Dermatol. 2010;3:32-43.

17. Sharquie KE, Al-Turfi IA, Al-Shimary WM. Treatment of acne vulgaris with $2 \%$ topical tea lotion. Saudi Med J. 2006;27:83-5.

18. Rivera AE. Acne scarring: a review and current treatment modalities. J Am Acad Dermatol. 2008;59:659-76.

19. Cotellessa C, Manunta T, Ghersetich I, Brazzini B, Peris K. The use of pyruvic acid in the treatment of acne. J Eur Acad Dermatol Venereol. 2004;18:275-8.

20. Monheit GD. Medium-depth chemical peels. Dermatol Clin. 2001;19:413-25, vii.

21. Levesque A, Hamzavi I, Seite S, Rougier A, Bissonnette R. Randomized trial comparing a chemical peel containing a lipophilic hydroxy acid derivative of salicylic acid with a salicylic acid peel in subjects with comedonal acne. J Cosmet Dermatol. 2011;10:174-8.

22. Smithard A, Glazebrook C, Williams HC. Acne prevalence, knowledge about acne and psychological morbidity in mid-adolescence: a community-based study. $\mathrm{Br} \mathrm{J}$ Dermatol. 2001;145:274-9.

23. Handog EB, Datuin MS, Singzon IA. Chemical peels for acne and acne scars in asians: evidence based review. J Cutan Aesthet Surg. 2012;5:239-46.

\begin{tabular}{l}
\hline MAILING ADDRESS: \\
Hassanain Al-Talib \\
Jalan Hospital Sungai Buloh, \\
Faculty of Medicine-UniversitiTeknologi MARA (UiTM) \\
47000 Sungai Buloh, Selangor, Malaysia \\
Email: hassanainiy@yahoo.com
\end{tabular}

How to cite this article: Al-Talib H, Hameed A, Al-khateeb A. Efficacy and safety of superficial chemical peeling in treatment of active acne vulgaris. An Bras Dermatol. 2017;92(2):212-6. 